\section{Cahiers de Narratologie}

Analyse et théorie narratives

$10.2 \mid 2001$

La voix narrative

\title{
De vive voix. Traitements polyphoniques dans Une année dans la vie de Gesine Cresspahl de Uwe Johnson
}

Helga Kaser

\section{(2) OpenEdition}

Journals

Édition électronique

URL : http://journals.openedition.org/narratologie/10191

DOI : 10.4000/narratologie. 10191

ISSN : 1765-307X

Éditeur

LIRCES

\section{Édition imprimée}

Date de publication : 1 janvier 2001

Pagination : 106-114

ISBN : 2914561032

ISSN : 0993-8516

\section{Référence électronique}

Helga Kaser, « De vive voix. Traitements polyphoniques dans Une année dans la vie de Gesine Cresspahl de Uwe Johnson », Cahiers de Narratologie [En ligne], 10.2 | 2001, mis en ligne le 01 janvier 2001, consulté le 11 juin 2020. URL : http://journals.openedition.org/narratologie/10191 ; DOI : https:// doi.org/10.4000/narratologie.10191 


\title{
DE VIVE VOIX. TRAITEMENTS POLYPHONIQUES DANS UNE ANNÉE DANS LA VIE DE GESINE CRESSPAHL DE UWE JOHNSON
}

\author{
Helga KASPER \\ Université de Saint-Etienne
}

\section{Introduction}

Le chef-d'œuvre de l'écrivain allemand Uwe Johnson (1934-1984), le roman Une année dans la vie de Gesine Cresspahl, est une tétralogie imposante d'environ 2000 pages. Terminé en 1983, il apparaît aujourd'hui comme un livre essentiel de (et sur) notre époque.

Gesine Cresspahl, l'hérö̈ne du roman, subit les mêmes vicissitudes dans son existence que son auteur : elle a connu le nazisme et les débuts de la R.D.A, qu'elle a quittée dans les années cinquante pour l'Allemagne de l'Ouest. En 1961, elle s'installe à New York où elle vit avec sa fille de dix ans, Marie. Jour après jour, sous la forme d'un journal allant du 20 août 1967 au 20 août 1968, Gesine raconte sa vie newyorkaise et ses souvenirs d'enfance et de jeunesse passées dans le Mecklembourg.

Aussi, l'ordrel narratif est-il structuré par la simultanéité entre le passé remémoré et le temps présent. D'un côté, la petite ville de Jerichow, proche de la Baltique, successivement sous le nazisme, le stalinisme et le socialisme est-allemand, de l'autre côté l'Amérique agitée par les remous de la guerre du Viêt-nam, les questions raciales et la violence des faits divers, perçus à travers le prisme froid du New York Times.

La linéarité narrative est ainsi abandonnée au profit d'un entrelacs de voix plurielles qui forment un tissu polyphonique. Cette polyphonie tient un rôle primordial dans l'économie narrative. Si l'on considère l'ensemble du texte,

${ }^{1}$ Ce terme est utilisé dans le sens de Gérard GENETTE, Figures III, Paris, 1972. 
trois types de traitement polyphonique se détachent très nettement, ayant chacun une fonction particulière. Lorsque Johnson met en scène la vox populi, il confère une fonction idéologique au traitement polyphonique, lui permettant un ancrage socioculturel du récit. Une deuxième fonction, que Gérard Genette appellerait métanarrative, apparaît dans l'articulation entre la voix du narrateur et celle du personnage principal : Qui raconte, qui parle ? Enfin, l'insertion du patois bas-allemand et des langues étrangères assure une fonction communicative et intertextuelle qui agit directement sur le lecteur ${ }^{2}$.

\section{Vox populi}

La superposition de plusieurs voix est un enjeu majeur dans Une année dans la vie de Gesine Cresspahl. Elle constitue un magma où se fonde le destin personnel, familial et collectif de quelque quatre cents personnages.

Par le jeu de la voix, Johnson nous offre l'image d'un univers social complexe, aux aspects historiques, linguistiques et idéologiques contrastés. A cet égard, deux scènes sont particulièrement frappantes.

La nuit du pogrom du neuf au dix novembre 1938, dit la Nuit de cristal, la mère de Gesine, Lisbeth Cresspahl, révoltée par l'assassinat d'une enfant juive, gifle un haut responsable national-socialiste. Le lendemain, elle est trouvée morte suite à un incendie. Lors de son enterrement, les habitants de Jerichow commentent les événements.

Not' Lisbeth.

Regarde la petite.

Quel malheur de mourir.

Ottje Stoffregen est déjà soûl. (...)

On met du bois dans le feu, on n'y met pas les pieds et les mains. Mourir en novembre, ça ne me plairait pas.

Le mois du brouillard.

Tout ça pour une gosse de Juifs. (GC II, p. 265)

2 A l'instar de Roman JAKOBSON et ses fonctions du langage (Essais de linguistique générale, Paris, 1963, p. 213-220), Gérard GENETTE, Figures III, p. 261-263, distribue les fonctions du narrateur selon les divers aspects du récit auxquels elles se rapportent. Notre classification s'y réfère. 
A New York, après l'assassinat de Martin Luther King, Gesine entend les propos suivants :

- Il se peut qu'on aille trop loin.

- Mettre les drapeaux en berne ! Ce n'était tout de même pas Kennedy !

- Les Noirs méritent d'être traqués bloc après bloc !

- C'était eux, si ça se trouve. (...)

- Croyez-vous qu'il n'y a vraiment eu aucun mort à Harlem hier soir ? On ne cherche qu'à nous rassurer. (GC II, p. 448)

Il est intéressant de constater que des situations aussi éloignées historiquement, telles la montée du nazisme en Allemagne ou l'opinion publique américaine à la fin des années soixante, sont rapprochées grâce au même traitement polyphonique. Johnson se sert de l'anonymat de ces voix, moyennant une écriture polyphonique, dans un but précis : sa critique politique et sociale va au-delà d'un système donné. Elle vise la déshumanisation de la voix, noyée dans un microcosme collectif. Tributaire de Brecht, la technique utilisée par Johnson entremêle inextricablement individu et société, sphère privée et sphère politique.

En réalité, dans ce passage, nous percevons un effacement des marques de l'énonciation au profit d'un phénomène communicatif de masse. Autrement dit, l'auteur semble vouloir laisser dans l'ombre l'origine de ces énonciations. Ce passage n'exhibe aucun auteur de la parole, ne comporte aucune mention d'origine. En effet, comme l'écrit très justement Oswald Ducrot :

(L)'existence d'une source et d'une cible sont parmi les qualifications que le sens donne (ou ne donne pas) à l'énonciation ${ }^{3}$.

Ici, la polyphonie naît d'une simple juxtaposition de plusieurs voix. Dans ce sens, elle est proche du collage. Cet effet d'énonciation ${ }^{4}$ anonyme, certes rudimentaire, s'inscrit néanmoins dans une recherche d'expression polyphonique évidente, assurant le fondu-enchaîné temporel et spatial entre

3 Oswald DUCROT, Le dire et le dit, Paris, 1984, p. 195.

4 Oswald DUCROT, Le dire et le dit, p. 174. Le terme effet d'énonciation est crée en analogie au terme courant effet de réel. 
le Mecklembourg des années trente et la ville de New York des années soixante.

\section{Un pacte étrange}

Un deuxième traitement polyphonique, plus raffiné encore, concerne non pas l'alternance de plusieurs voix, mais l'interférence de deux voix au sein d'un même énoncé, notamment celles du narrateur et de Gesine. Ces images doubles, et souvent troubles, de l'énonciation sont porteuses de sens. La construction d'une seule ou de plusieurs instances d'énonciation pour un seul énoncé modifie profondément la signification de la phrase.

Considérés comme les deux pivots constitutifs de la fiction, le narrateur et le personnage principal ont conclu un pacte étrange dans Une année dans la vie de Gesine Cresspahl. Johnson fait coexister deux systèmes énonciatifs autonomes. Or, trois particularités troublent la dissociation habituelle entre narrateur et personnage principal.

Tout d'abord, l'auteur abolit la frontière entre l'univers extradiégétique et intradiégétique. L'auteur Johnson apparaît sous son véritable nom dans le roman, relate des événements qui lui sont réellement arrivés et il cherche même conseil auprès de l'héroïne Gesine.

Oui, si seulement je t'avais demandé conseil, Mrs Cresspahl.

A l'époque, nous ne nous parlions pas encore, M. Johnson. (GC I, p. 289)

C'est dire combien, pour cet auteur, le monde réel et le monde fictif sont intimement liés. Le sens et la légitimation du roman semblent se construire dans ce rapport énonciatif d'interlocution et d'échange discursif.

Mais qui raconte, Gesine?

Nous deux. Tu t'en aperçois bien, Johnson. (GC I, p. 288)

L'auteur nous propose un dédoublement du narrateur, une relation intrinsèque de coexistence tout au long du livre entre Gesine et Johnson.

Plus originalement encore, l'auteur semble perdre le pouvoir absolu sur ces personnages. C'est comme si le personnage central, Gesine, transférait le pouvoir de narration à Johnson. 
Maints rappels à l'ordre de la part de Gesine obligent l'auteur à modifier le cours de son récit.

(E)lles s'étaient retrouvées sur la crête du Heidelberg (...), où s'ouvre aussi une échappée sur l'île du lac et sur la campagne qui s'élève doucement derrière l'eau avec son décor chiche d'arbres et de toits, lumineux quand le soleil a pu à l'instant chasser des nuages de pluie lourds ; ce spectacle, qu'il me soit présent à l'heure de mon

Que cela pèse si lourd sur toi, on s'en fiche, camarade écrivain! Ecris-le donc! Nous pouvons d'ailleurs en terminer avec ton livre aujourd'hui même! A toi d'imaginer ce que la mort nous inspire.

agonie. (GC IV, 426)

Le pacte énonciatif, tel que Johnson le conçoit, consiste dans la provocation, l'interrogation et l'écoute mutuelle entre l'écrivain et son personnage. Il en résulte un jeu complexe de deux voix, de deux intentions croisées, complémentaires ou contradictoires. Uwe Johnson revendique un principe de discontinuité, alternant rapidement les différents niveaux narratifs ainsi que des instances énonciatives diverses. Avec Mikhaïl Bakhtine, nous pouvons qualifier de construction polyphonique ou hybride au sens restreint du terme un énoncé qui :

d'après ses indices grammaticaux (syntaxiques) et compositionnels, appartient au seul locuteur, mais où se confondent, en réalité, deux énoncés, deux manières de parler, deux styles, deux « langues », deux perspectives sémantiques et sociologiques 5 .

Cet espace particulier de la double énonciation surdétermine tout le roman. De ce point de vue, l'exemple suivant, une lettre adressée à un ami, citoyen de la R.D.A. est particulièrement stimulant :

Nous prenons le nous pour te parler afin que tu sois à même d'utiliser comme prétexte l'opacité du nombre ; qu'en revanche, en cas de réserve, tu puisses supposer et faire inscrire au procès-verbal que quelqu'un parle ici pour d'autres auprès de qui il/elle a agi afin de t'attirer la

5 Mikhail BAKHTINE, Esthétique et théorie du roman, Paris, 1978, p. 125 sq. 
confiance et déjà le respect de ton nom. Choisis, tu sauras bien. (GC IV, p. 249)

Ici, l'origine de l'énonciation, clin d'œil métatextuel, est sciemment camouflée sous le pronom personnel de la première personne du pluriel. A qui impute-t-on la responsabilité de cet énoncé ? La réponse restera ambivalente, car cet effet d'énonciation double, également reflété dans l'agencement de deux pronoms personnels il/elle fait imploser la frontière entre deux systèmes énonciatifs autonomes. Il semblerait que l'on puisse parler de la scénographie narrative d'un espace ambigu, étant par là même irréductible et paradoxal. Johnson nous suggère une pluralité de responsables donnés pour distincts et irréductibles. Dans ce dialogue interne, les deux voix sont inextricablement soudées. Un décodage double semble s'imposer. L'auteur ne nous invite-t-il pas à analyser un discours biographique ? Ce genre littéraire, ne serait-il pas hybride à son tour, une sorte de fiction autobiographique?

Nous sommes soumis pendant cinq jours à un travail de huit heures, t'en souvient-il ; je te le dis en confidence : c'est plus. Resteraient les week-ends. Nous aurions pu sans doute nous décharger sur un camarade écrivain, nous l'avons sous la main, et même dans la nôtre ; comme ta proposition me désignait, il fallut en rester à moi, entre nous. (GC IV, p. 250sq)

Sur le plan strictement narratif, cette oscillation entre le je et le nous, ou bien entre la première et la troisième personne du singulier, souligne une marque de distanciation plus au moins grande par rapport au narré. Il est d'ailleurs intéressant de noter qu'une certaine emphase se fait entendre dès que le je entre en jeu. La distanciation, l'emphase et élément autobiographique semblent être des effets d'énonciation rendus possibles grâce au dédoublement énonciatif. La voix d'autrui s'infiltre subrepticement dans un discours donné, un léger décalage linguistique fait retentir une sorte d'étrangeté sémantique, et l'effet d'énonciation en est durablement transformé.

Ainsi, à l'aide du jeu subtil des énonciateurs multipliés avec virtuosité, la littérature de Johnson est-elle la mise en scène permanente d'une fragilité que font ressentir la rupture de l'illusion réaliste et le dévoilement de la fiction. 


\section{Fonctions du patois et des langues étrangères}

Le patois bas-allemand, fréquemment parlé dans le Mecklembourg, joue un rôle important dans le dispositif énonciatif. D'abord associé au caractère oral de certains dialogues, le patois se révèle également un outil puissant de distanciation dans le récit hétérodiégétique pris en charge par le narrateur. Aussi, l'expression sich einen weissen Fuss machen, qui ressemble à l'expression française montrer patte blanche, est-elle transposée du bas-allemand en allemand standard. Cette tournure est incompréhensible pour un germanophone ne maîtrisant pas ce patois. Le lecteur non averti reste perplexe, trouvant cette expression aussi bien dans un discours direct de Gesine que dans le récit au sens strict du terme où le narrateur relate des événements du passé6. Évidemment, la traduction du patois est un problème épineux et la traduction d'Anne Gaudu, excellente par ailleurs, ne retient que le sens premier de l'expression, se faire une vertu ou entrer dans les bonnes grâces de quelqu'un. Le moment déconcertant qui saisit le lecteur germanophone, ne comprenant plus sa propre langue, est effacé. Est effacée également une source de polyphonie. Est-ce vraiment le narrateur qui raconte ou, au contraire, n'est-ce pas la voix de Gesine qui se fait entendre d'une manière contrapuntique ?

De même, dans une lettre du comité central du parti communiste tchèque, datant du 26.7.1968, réponse officielle à la résolution de la conférence de Varsovie du 15.7.1968, qui est citée presque in extenso en allemand dans le roman, se glissent des mots en patois bas-allemand ${ }^{7}$. La prise de conscience de cette double énonciation permet seule d'apprécier au lecteur les effets d'énonciation complexes d'ironie et de critique subtiles. Par le biais du patois, la

6 Uwe JOHNSON, Jahrestage. Aus dem Leben der Gesine Cresspahl, Frankfurt am Main, 1993, p. 835 et p. 1855.

7 Le 19 juillet 1968 on trouve la phrase suivante en allemand : Gerade jetzt ist es bannig schwierig. En patois bas-allemand, le mot bannig signifie particulièrement. Dans la traduction française, le terme bien semble indiquer l'intrusion d'un style parlé : Juste en ce moment tout est bien difficile. (JOHNSON Uwe, Une année dans la vie de Gesine Cresspahl, tome 4, p. 195). 
citation se transforme en simulacre au lieu d'être présentée comme la répétition du signifiant du discours cité.

Outre une fonction communicative, polyphonique ou ironique, le patois bas-allemand assume dans ce roman un rôle esthétique. Johnson prend la langue allemande à rebroussepoil en transposant des syntagmes dialectaux en allemand standard. Plus originalement encore, il enrichit l'allemand et s'inscrit par là même dans une tradition esthétique, allant de Theodor Fontane à Thomas Mann. Prenons par exemple l'expression er war sich nichts vermutend gewesen ${ }^{8}$, il ne s'était jamais douté de rien (GC I, 204). Elle est d'une connotation archaïque particulièrement stylisée, voire maniérée en allemand. On est porté à croire que Johnson invente de nouvelles formes grammaticales. D'ailleurs, des critiques ont reproché à Johnson de ne pas savoir écrire l'allemand. Or, c'est une expression courante dans l'œuvre de Thomas Mann, utilisée par exemple dans la nouvelle Mario et le magicien, publié en $1930^{\circ}$. Force est de constater que ce type de polyphonie, fondé sur le patois, gagne une dimension supplémentaire grâce à ces allusions intertextuelles.

Des langues étrangères comme le russe, le tchèque, l'italien ou le français sont également très présentes dans cette œuvre. Seul l'emploi de l'anglais se distingue par un traitement hybride ou polyphonique, qui se traduit par un enchevêtrement de l'anglais et de l'allemand, aussi bien sur le plan lexical que syntaxique. Au niveau lexical, cette particularité d'une polyphonie bilingue, prononcée par une seule personne se manifeste dans le terme Wildernis ${ }^{10}$, contrées sauvages, néologisme issu du mot allemand Wildnis et du mot anglais wilderness. Quant à la syntaxe, le passage suivant illustre assez bien la polyphonie bilingue au sein d'un seul énoncé. Gesine rentre à la maison :

Et les derniers pas puis devant la porte de l'appartement, il ne reste plus du cadeau que le désir de le faire, and saying some thing (sic) stupid like : Tu sais, Marie. (GC III, p. 313)

8 Uwe JOHNSON, Jahrestage, p. 180.

9 Thomas MANN, Die Erzählungen, Frankfurt am Main, 1986.

10 Uwe JOHNSON, Jahrestage, p. 1646. 
La citation d'une chanson de Frank Sinatra, Strangers in the night, n'est pas un jeu gratuit. Au contraire, elle introduit une valeur sémantique supplémentaire. En effet, la polyphonie y est conçue comme une suspension, l'émotion est mise à distance et se mue en citation musicale. Comme si Gesine voulait tourner en dérision ses propres pensées et sentiments. Ici, la polyphonie permet-elle à l'auteur de s'inscrire en faux contre sa propre énonciation, tout en l'accomplissant? Quoi qu'il en soit, à travers le langage, la relation entre l'individu et le monde nous est présentée comme radicalement distanciée.

L'utilisation systématique des langues étrangères et du patois bas-allemand dépasse largement l'horizon d'attente du lecteur. Beaucoup de bruit, pour reprendre le terme de Iouri Lotman, complexifie la communication. Contre toute attente, ce sont des effets de réel, comme le patois et les langues étrangères, qui sont souvent incompréhensibles au prime abord et, par conséquent, faussement interprétés comme des effets de fiction. Cette hétérogénéité idiomatique crée par conséquent une oscillation permanente entre l'effet de réel et l'effet de fiction.

Qui parle, qui écrit dans Une année dans la vie de Gesine Cresspahl? En effet, la polyphonie, au cœur du texte romanesque, privilégie une parole dense et riche de sens. Car le discours des personnages n'est jamais simplement transmis ou reproduit. Il est représenté avec art. Partout dans le récit des paroles, la voix du narrateur se fait entendre avec brio, aussi bien dans le discours direct que dans des documents officiels cités. Inversement, le récit des événements semble affecté à son tour par le style oral des personnages. Toute la dynamique de la syntaxe est marquée par la parataxe et l'anacoluthe, caractéristiques de la langue parlée en allemand. Ce principe de porosité entre langue écrite et langue parlée ou entre des langues différentes, bref, entre l'altérité et l'identité, s'avère fondamental pour l'esthétique de Uwe Johnson.

\section{Conclusion}

Le plurilinguisme attire notre attention sur le matériau esthétique de base de toute œuvre littéraire, à savoir la langue. Le 
roman Une année dans la vie de Gesine Cresspahl est résolument métalinguistique et métatextuel, une sorte de précis de l'histoire de la langue allemande. C'est avant tout un roman sur le langage, sa beauté et sa manipulation possible.

Le contrepoids à ces idéologies réside dans la structure dialogale de l'œuvre. La polyphonie, au cœur du discours romanesque de Uwe Johnson, s'inscrit en faux contre le discours monologique et hiératique de toute autorité. Comme le souligne fort justement Michel Aucouturier :

Le roman, c'est la vie triomphant de l'idéologie ${ }^{11}$.

11 Michel AUCOUTURIER, «Mikhail Bakhtine philosophe et théoricien du roman (préface) ", in Mikhail BAKHTINE, Esthétique et théorie du roman, Paris, 1978, p. 19. 